Direct oxygen imaging in titania nanocrystals

This content has been downloaded from IOPscience. Please scroll down to see the full text. 2012 Nanotechnology 23335706

(http://iopscience.iop.org/0957-4484/23/33/335706)

View the table of contents for this issue, or go to the journal homepage for more

Download details:

IP Address: 148.234.36.245

This content was downloaded on 28/01/2016 at 17:11

Please note that terms and conditions apply. 


\title{
Direct oxygen imaging in titania nanocrystals
}

\author{
Weigang Lu' ${ }^{1}$, Britain Bruner ${ }^{1}$, Gilberto Casillas ${ }^{2}$, Sergio Mejía-Rosales ${ }^{3}$, \\ Patrick J Farmer ${ }^{1,4}$ and Miguel José-Yacamán ${ }^{2,4}$ \\ ${ }^{1}$ Department of Chemistry and Biochemistry, Baylor University, Waco, TX 76796, USA \\ ${ }^{2}$ Department of Physics, University of Texas at San Antonio, San Antonio, TX 78249, USA \\ ${ }^{3}$ Center for Innovation and Research in Engineering and Technology, and CICFIM-Facultad de Ciencias \\ Físico-Matemáticas, Universidad Autónoma de Nuevo León, San Nicolás de los Garza, NL 66450, \\ Mexico \\ E-mail: patrick_farmer@baylor.edu and miguel.yacaman@utsa.edu
}

Received 24 April 2012, in final form 6 July 2012

Published 3 August 2012

Online at stacks.iop.org/Nano/23/335706

\begin{abstract}
Recently, rutile nanotwins were synthesized using high temperature organic solvent methods, yielding two kinds of common high-quality rutile twinned nanocrystals, (101) and (301) twins, accompanied by minor rutile nanorods (Lu et al 2012 CrystEngComm 14 3120-4). In this report, the atomic structures of the rutile and anatase nanocrystals are directly resolved with no need for calculation or image simulation using atomic resolution STEM techniques. The locations of the oxygen rows in the rutile twins' boundaries are directly determined from both HAADF images and ABF images. To the best of our knowledge, this is the first time oxygen columns have been distinguished in rutile twin boundaries using HAADF and BF imaging.

S] Online supplementary data available from stacks.iop.org/Nano/23/335706/mmedia
\end{abstract}

(Some figures may appear in colour only in the online journal)

\section{Introduction}

Titania is an important material with numerous applications in the fields of catalysis, photocatalysis, new energy sources and environmental protection [2-4]. The catalytic activity of $\mathrm{TiO}_{2}$ depends on its crystal structure, morphology, surface facets, particle size and surface chemistry. Among the three titania polymorphs (rutile, anatase, and brookite), anatase has the highest catalytic activity, and hence most catalytic applications such as dye-sensitized solar cells [2], and water splitting [3] are based on anatase nanostructures. As a model system of transitional metal oxides, atomic level catalysis studies are typically conducted on the rutile $\mathrm{TiO}_{2}(110)$ surface due to its excellent electronic properties and well understood surface chemistry. Defects such as oxygen vacancies (OVs) in titania may dominate its electronic and chemical properties since OVs may act as the primary absorption sites for catalytic reactions $[5,6]$. Rutile is the preferred polymorph among the three common titania polymorphs because it has the lowest

4 Authors to whom any correspondence should be addressed. bulk Gibbs free energy [7]. The anatase and brookite phases convert to rutile at elevated temperature [8-10].

Progress in electron microscopy has allowed material characterization down to atomic level during the past two decades [11-17]. Innovations in aberration-corrected (Cs) transmission electron microscope (TEM) and scanning transmission electron microscope (STEM) sub-ångström resolution imaging have been achieved. Using a Cs-corrected HRTEM, Yoshida et al [18] reported direct observation of oxygen atoms and magnetic structure caused by oxygen vacancies in rutile. In their observation, two oxygen columns and one titanium row formed atomic triplets, and the improvement in spatial resolution after the Cs correction enabled the distinction of the oxygen columns from the titanium rows. Shibata et al [19] used a high voltage electron microscope which operated at $1250 \mathrm{kV}$ combined with a high-angle annular dark field scanning transmission electron microscope (HAADF-STEM) to study the reconstruction onthe $\mathrm{TiO}_{2}(110)$ surface. Their observations showed that the interstitial $\mathrm{Ti}$ atoms are involved in the $\mathrm{TiO}_{2}(110)$ surface reconstruction, which is consistent with the model proposed by Park et al [20] based on scanning tunneling microscopy 
observations and DFT calculations. In a comparison study using high-angle annular dark field (HAADF) and annular bright field $(\mathrm{ABF})$ images recorded simultaneously, the oxygen rows in titania can be resolved from the bow-tie patterns in the $\mathrm{ABF}$ image; the vague strips between titanium rows suggest the approximate positions of the oxygen columns in the HAADF image [21]. Also, De Caro et al [22] developed a new phase-retrieval algorithm that allows the crystal structure of titania nanocrystals to be determined at the sub-ångström level in HRTEM experiments without the need for aberration correction. For example, the oxygen positions in anatase nanocrystals were revealed by 'extracting' useful information from diffractive images and conventional high resolution TEM images.

We have previously developed an approach to produce rutile nanotwins using high temperature organic solvent methods: two kinds of common high-quality rutile twinned nanocrystals, (101) and (301) twins, accompanied by minor rutile nanorods [1]. In this paper, the atomic structures of the rutile and anatase nanocrystals are directly resolved with no need for calculation or image simulation using atomic resolution STEM techniques. The locations of the oxygen rows in the rutile twins' boundaries are directly determined from both HAADF images and ABF images. To the best of our knowledge, this is the first time that oxygen columns have been distinguished in rutile twin boundaries using HAADF and BF imaging.

\subsection{Experimental chemicals}

Oleylamine (90\%), oleic acid (90\%), hexane and anhydrous ethanol were bought from Aldrich or Acros, and used as received, without purification.

\subsection{Synthesis of rutile nanocrystals}

In a typical synthesis of rutile nanocrystals, $5 \mathrm{ml}$ of oleylamine was mixed with $1 \mathrm{ml}$ of oleic acid in a three necked flask. A $0.25 \mathrm{ml}$ portion of $1 \mathrm{M} \mathrm{TiCl}_{4}$ in toluene was injected into the mixture. The mixture was heated for $30 \mathrm{~min}$ to remove low boiling point substances at $180^{\circ} \mathrm{C}$, then the temperature was increased to $350^{\circ} \mathrm{C}$ under vigorous stirring. The color of the mixture changed from reddish to gray as the reaction took place; the reaction was quenched by adding hexane into the flask. The product was isolated by adding a sufficient amount of ethanol, separated with centrifugation and washed several times with a hexane-alcohol solvent to get $\mathrm{TiO}_{2}$ nanorods.

\subsection{STEM characterizations}

The atomic structures of the titania nanocrystals were characterized with a JEOL JEM-ARM200F electron microscope. The $\mathrm{TiO}_{2}$ nanocrystals made from organic solvents were usually covered with a layer of capping ligands, but the removal of this organic layer by placing the samples under a $10 \mathrm{~min}$ beam shower is straightforward. STEM images were simultaneously recorded in both HAADF and BF modes with the microscope operating at $200 \mathrm{kV}$. The probe correction was performed through a dodecapole corrector (CEOS $\mathrm{GmbH}$ ) aligned with CESCOR software, to finally obtain a twelve-fold ronchigram with a flat area of $50 \mathrm{mrad}$. The HAADF images were obtained by setting the annular detector lower and higher scattering semi-angles to 68 and $280 \mathrm{mrad}$ respectively, easily satisfying the requirement for the detector to eliminate contributions from unscattered or low-angle scattered electrons. Images were commonly recorded for 10-16 s. The data were collected and processed using Gatan Digital Micrograph software.

\subsection{Simulations}

Atomistic models of the samples were prepared based on the known rutile structure and emulating the features of the structures imaged in the STEM micrograph, in particular reproducing the 101 and 301 twins likely to be present in the real structures. These models were first used to generate images where the intensity in each pixel was calculated considering a contribution of $Z^{1.7}$, with the purpose of estimating the intensity profile expected in an STEM micrograph of a sample with the same structure and orientation as that of the model. Both the ball and stick models and the images produced in this way are included in the supplementary information (available at stacks.iop.org/Nano/ $23 / 335706 /$ mmedia), where it can be verified that the real and simulated intensity profiles are quite similar, which we consider as evidence of the similarity between the models and the real structures. After this test, STEM simulations based on the more precise multislice method were performed using the xHREM suite, which is based on the version of the FFT multislice method by Ishizuka [23]. The settings used in the simulation were kept as close as possible to those used in the generation of the real micrographs: the simulated electron beam was $200 \mathrm{kV}$, with a resolution close to $0.1 \mathrm{~nm}$ and a defocus of $10 \mathrm{~nm}$. Since the FFT multislice method is computationally expensive, the generation of a simulated STEM image of the whole structure was an impractical task, and thus the images obtained by this method were restricted to small regions.

\section{Results and discussion}

Scanning transmission electron microscopes (STEMs) use a very small diameter electron beam to scan samples. STEM images of different modes are formed by collecting electrons with detectors at different positions. In contrast, HAADF images are formed by collecting electrons using a detector with a large inner radius, and these HAADF images show little or no diffraction effects, with the peak intensity approximately proportional to the atomic number [24]. These properties make it a robust imaging mode for the identification of heavy elements, but light elements are usually uncharacterized due to their relatively weak signals [25]. In the imaging of oxygen atoms, the negative spherical aberration technique, developed by Urban et al, not only allows atomic resolution imaging of oxygen, but also gives the oxygen vacancy concentration in the oxide twin boundaries $[26,27]$. In recent years, the annular 

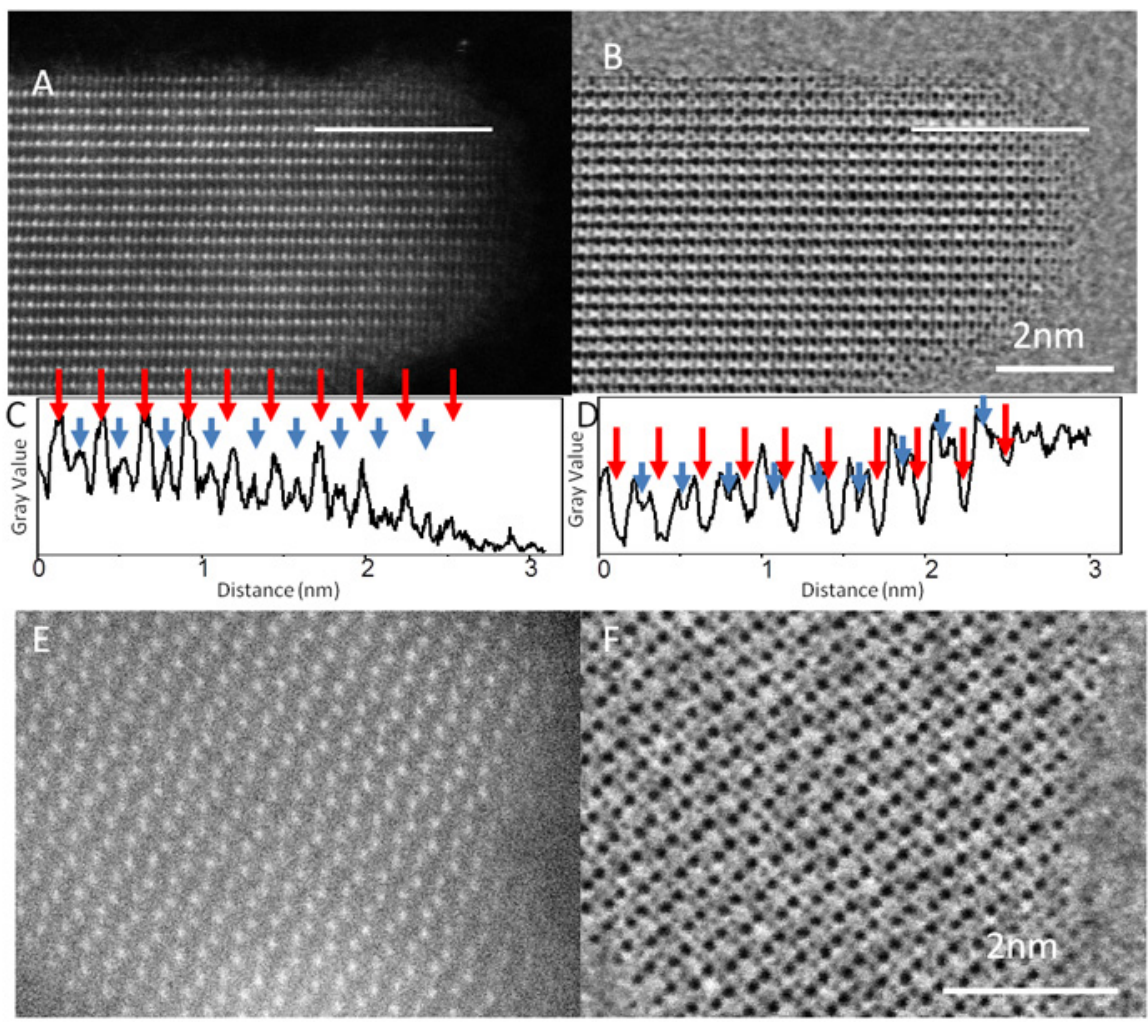

Figure 1. HAADF-STEM and bright field raw images of rutile (110) and anatase (100) surfaces. (A) The HAAADF rutile (110) structure viewed along the [110] projection. (B) The BF image simultaneously recorded. In this image, the brighter features correspond to Ti-O rows and the dark features correspond to Ti rows. (C), (D) Bright field images of the rutile (110) surface. In the bright field image, Ti-O and Ti row oxygen can be identified. (E) The HAADF image of the anatase (100) surface. The bright features correspond to Ti rows. (F) The bright field image of the anatase (100) surface. The larger black spots are from Ti rows and the smaller dark features are from $\mathrm{O}$ rows.

bright field $(\mathrm{ABF})$ imaging technique has been successfully used to image light atoms such as nitrogen, boron [28], and lithium [29-31]; even the imaging of the lightest element, hydrogen, has been achieved recently [32, 33].

Figure 1 shows unprocessed STEM images of rutile and anatase nanocrystals. Figures $1(\mathrm{~A})$ and (B) are simultaneously recorded HAADF and BF images of rutile $\langle 110\rangle$. Figures $1(C)$ and (D) are the intensity profiles of the white lines in figures $1(\mathrm{~A})$ and $(\mathrm{B})$, respectively. In the HAADF image of rutile $\langle 110\rangle$ (figure $1(\mathrm{~A})$ ), the positions of the oxygen rows between $\mathrm{Ti}$ only rows are not resolved; two kinds of Ti-containing atomic columns can clearly be discriminated. The brighter contrast corresponds to Ti-O rows, the weaker contrast corresponds to $\mathrm{Ti}$ rows. The larger peaks in the intensity line profile are from $\mathrm{Ti}-\mathrm{O}$ rows (indicated with red arrows), the smaller peaks (labeled with blue arrows) are from Ti only rows. In the bright field image of rutile $\langle 110\rangle$ (figure 1(B)), the larger dark contrast corresponds to Ti-O rows and the smaller dark contrast corresponds to Ti rows. The gray strips between Ti rows reveal the positions of oxygen rows, but the two oxygen rows cannot be distinguished. In the intensity line profile of the bright field image, the larger troughs are from Ti-O columns and the smaller ones are from $\mathrm{Ti}$ rows. In the HAADF image of anatase (100), the titanium rows are revealed as bright pairs of spots, the oxygen columns between the titanium pairs not being visible; in the simultaneously recorded bright field, the larger dark contrast corresponds to Ti rows and the smaller dark contrast corresponds to oxygen rows which are clearly visible [19]. Figures 2(A) and (D) present the crystals ball models of rutile from [110] direction and anatase from the [1] direction. In the rutile model, there are two titanium-containing columns (referred to as $\mathrm{Ti}-\mathrm{O}$ columns and $\mathrm{Ti}$ only columns). The positions of the oxygen only columns are between the $\mathrm{T} i$ only columns. In the anatase model, there are pairs of Ti-O columns and pairs of oxygen only columns.

Noise reduction is crucial for improving the resolution of the STEM images. There is noise from the counting statics and read out noise from the imaging although many improvements in STEM hardware have been made. The coated amorphous carbon film on the TEM grids may increase the noise level as well. Removal of such noise sources in STEM-HAADF images can improve their contrast and resolution. The processed STEM images of rutile (110), anatase (001) and their ball models are presented in figure 2. Figures 2(B), (C), (E), and (F) present processed HAADF and $\mathrm{BF}$ images of the rutile and anatase nanocrystals in figure 1. The signal to noise ratio in all the images was improved by using an average filter. The contrast of the oxygen only columns in the rutile BF image gives a much clearer possible position of the oxygen columns. Comparing the HAADF and BF imaging of rutile and anatase nanocrystals, atomic resolution images of titanium-containing columns can readily be achieved in both imaging modes, while BF imaging 

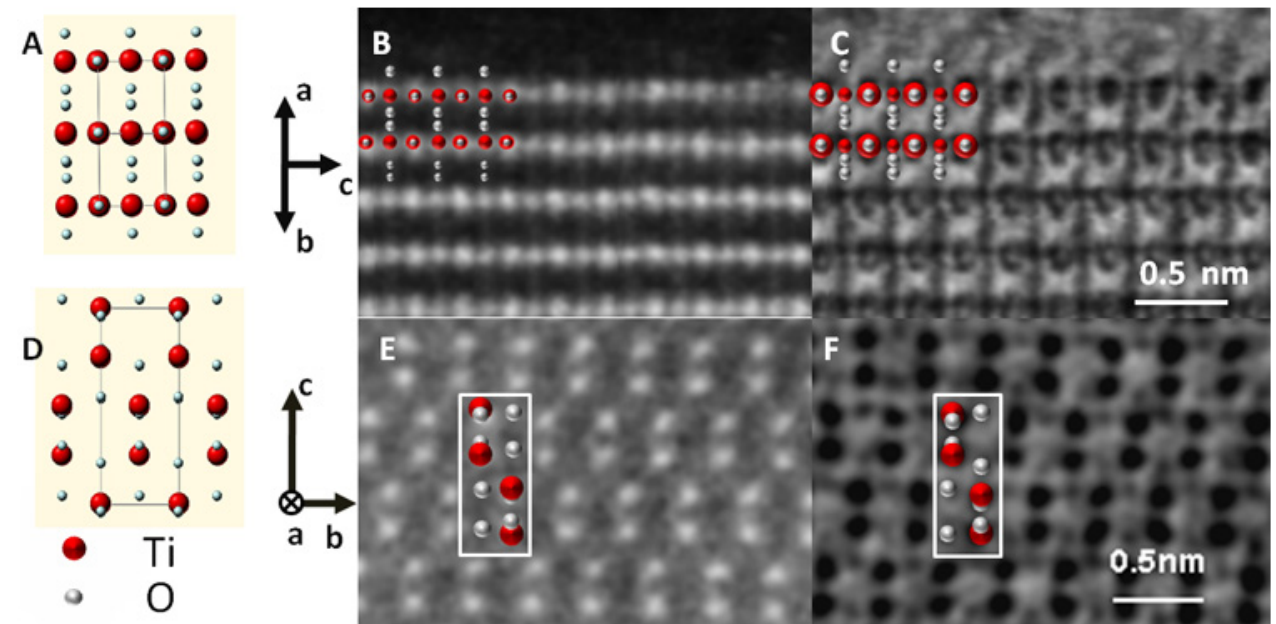

Figure 2. Processed atomic resolution HAADF-STEM and bright field images of rutile (110) and anatase (100) surfaces. (A), (D) Ball models of rutile (110) structure viewed from the $\langle 1 \overline{1} 0\rangle$ direction and anatase (100) structure viewed from the $\langle 010\rangle$ direction. (B), (C) Processed HAADF and BF images of the rutile (110) surface. In the HAADF image, the brighter features correspond to Ti-O rows and the darker features correspond to Ti rows. In the BF image of the (110) surface, Ti-O and Ti rows can be identified, with oxygen revealed as strips. (E), (F) Processed HAADF and bright field images of the anatase (100) surface. In the HAADF image, the bright features correspond to Ti rows. In the BF images, the larger dark spots are from Ti rows and the smaller dark features are from $\mathrm{O}$ rows.

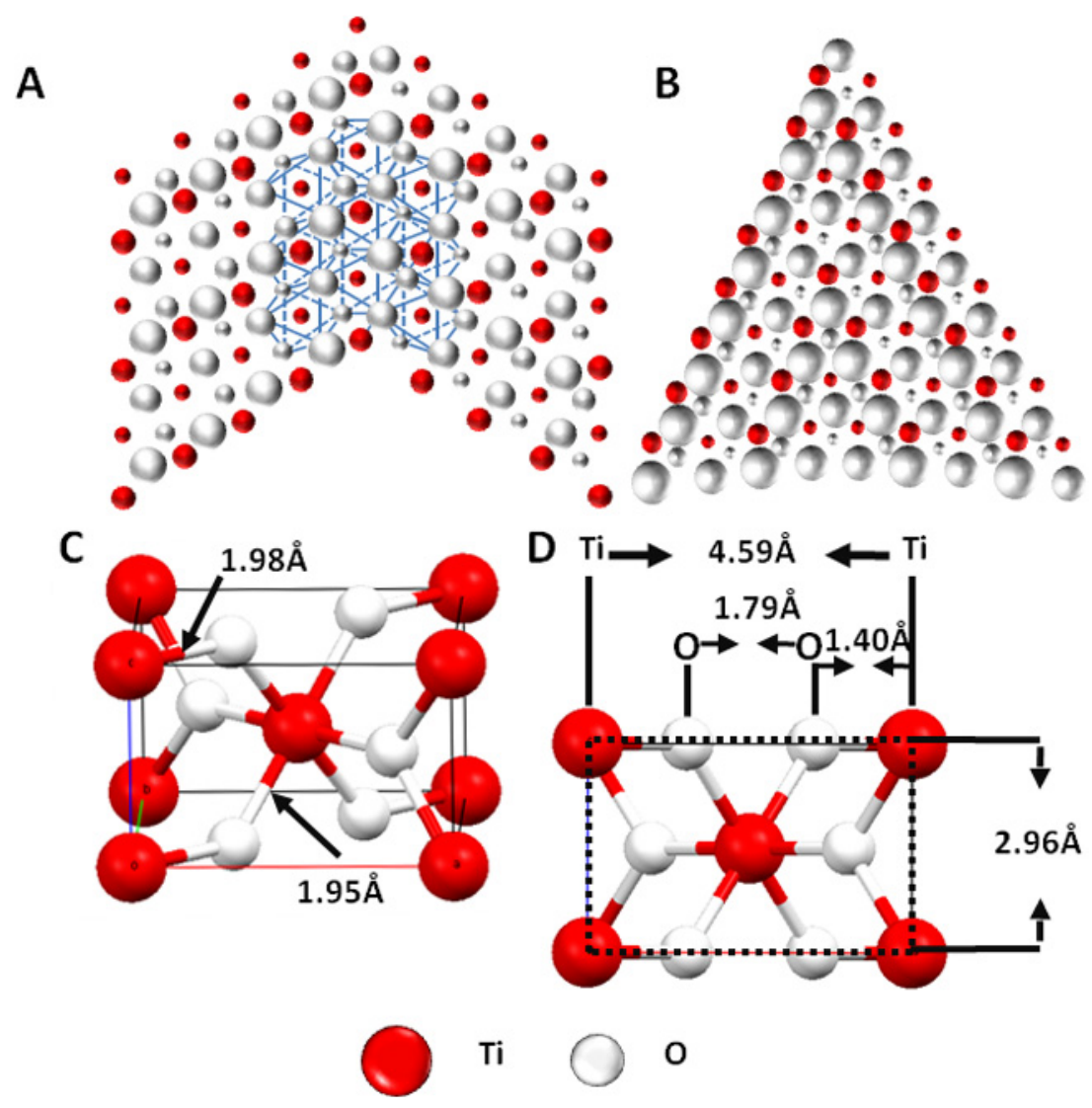

Figure 3. Model structure of rutile twins' boundaries and the rutile unit cell. (A) Atomic configuration of the (101) twin boundary, (B) atomic configuration of the (301) twin boundary. The sphere size indicates the depth along the [010] direction. (C) The unit cell of rutile crystal. (D) The rutile unit cell viewed along the [010] direction. The red and white spheres represent Ti columns and oxygen rows respectively.

provides a better resolution in the identification of oxygen rows for both rutile and anatase nanocrystals.

Twinning is a common phenomenon in crystal and thin film growth, and the peculiarities of the atomic structure of twinned materials may impact the optical, electronic and mechanical properties of the crystal. Rutile sometimes displays well defined elongated prismatic shapes with twinning in the form of elbows and hearts, as well 


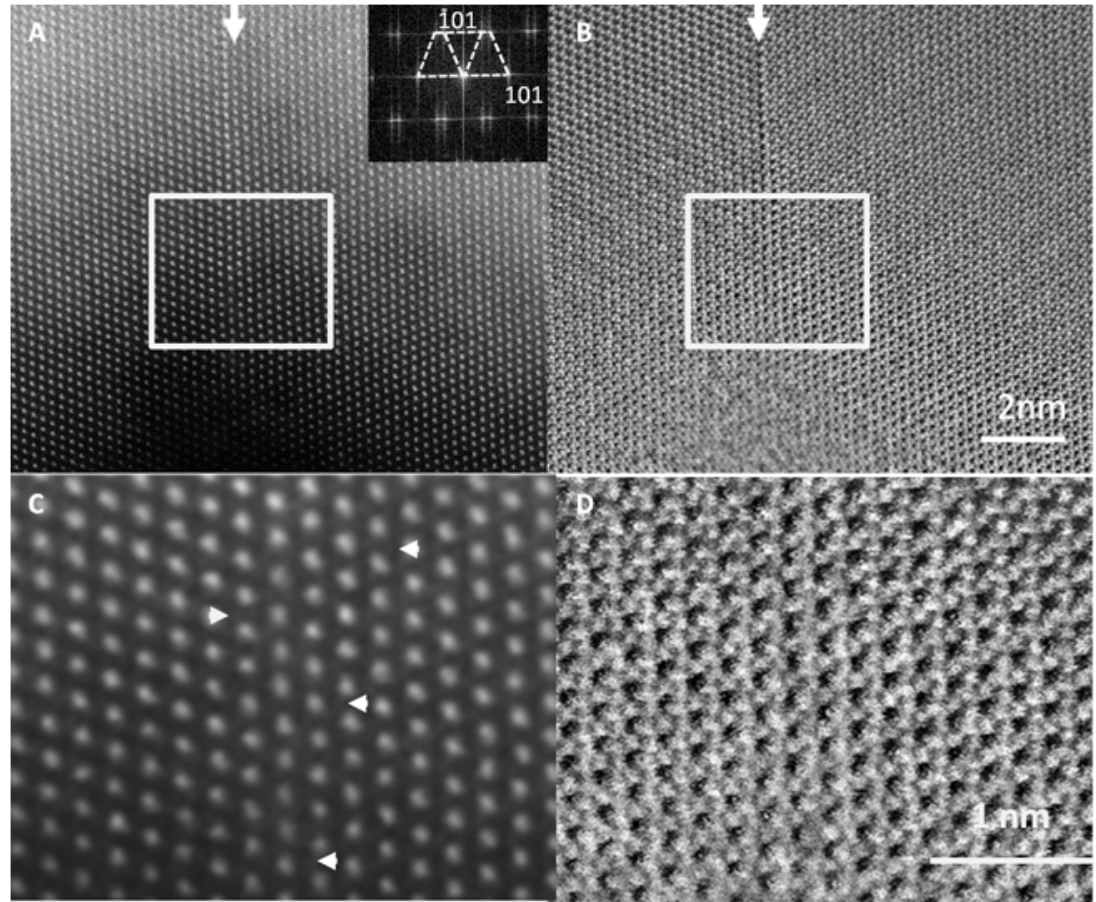

Figure 4. Atomic resolution HAADF and BF images of the rutile (101) twin boundary. (A) Large area HAADF image. The inset FFT pattern of the image shows symmetric patterns of (301) structures. (B) The BF simultaneously recorded. (C) An enlargement of the rectangle area in (A). The bright features are Ti rows, and oxygen columns between Ti rows can be resolved (the oxygen positions are highlighted with white arrows). (D) An enlargement of the rectangle area in (B). Both titanium rows and oxygen can be resolved.

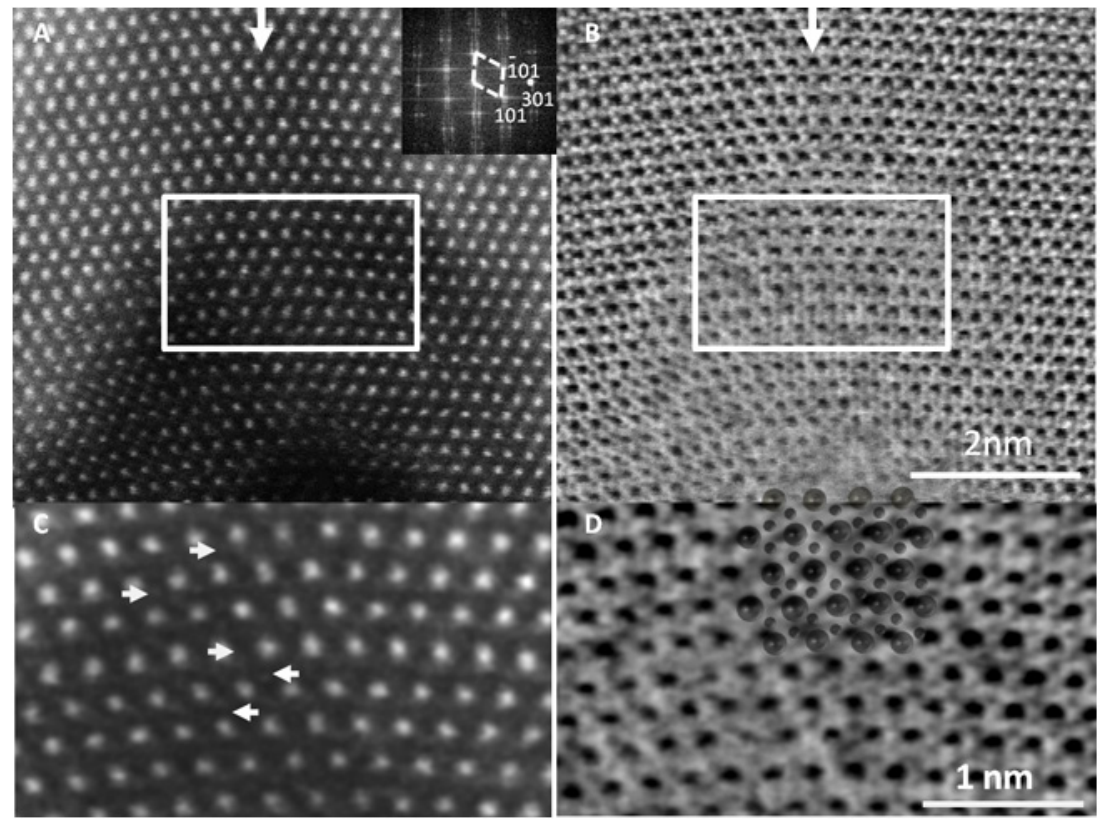

Figure 5. Atomic resolution HAADF and BF images of the rutile (301) twin boundary. (A) The unprocessed large area HAADF image. The inset FFT pattern of the image shows symmetric patterns of (301) structures. (B) The BF images simultaneously collected. (C) An enlargement of the rectangle area in (A). The bright features are Ti rows, and oxygen columns between Ti rows can be resolved (the oxygen positions are highlighted with white arrows). (D) An enlargement of the rectangle area in (B). The larger black spots are Ti rows and the smaller dark spots between Ti rows are O columns.

as reticulated twins [34]. The atomic structures of (101) twins and (301) twins have been proposed from theoretical calculations and conventional HRTEM [35-37], but the positions of the oxygen rows were not determined because the lattices obtained in the images were mainly produced by the metal columns. In (101) twins, the twin plane consists of Ti atoms shared by two twin domains, and the sublattices of $\mathrm{Ti}$ atoms are mirror symmetric. The oxygen atoms around the $\mathrm{Ti}$ atoms are not mirror symmetric, but have $1 / 2\langle 111\rangle$ displacement from mirror symmetric positions (figure $3(\mathrm{~A})$ ). 


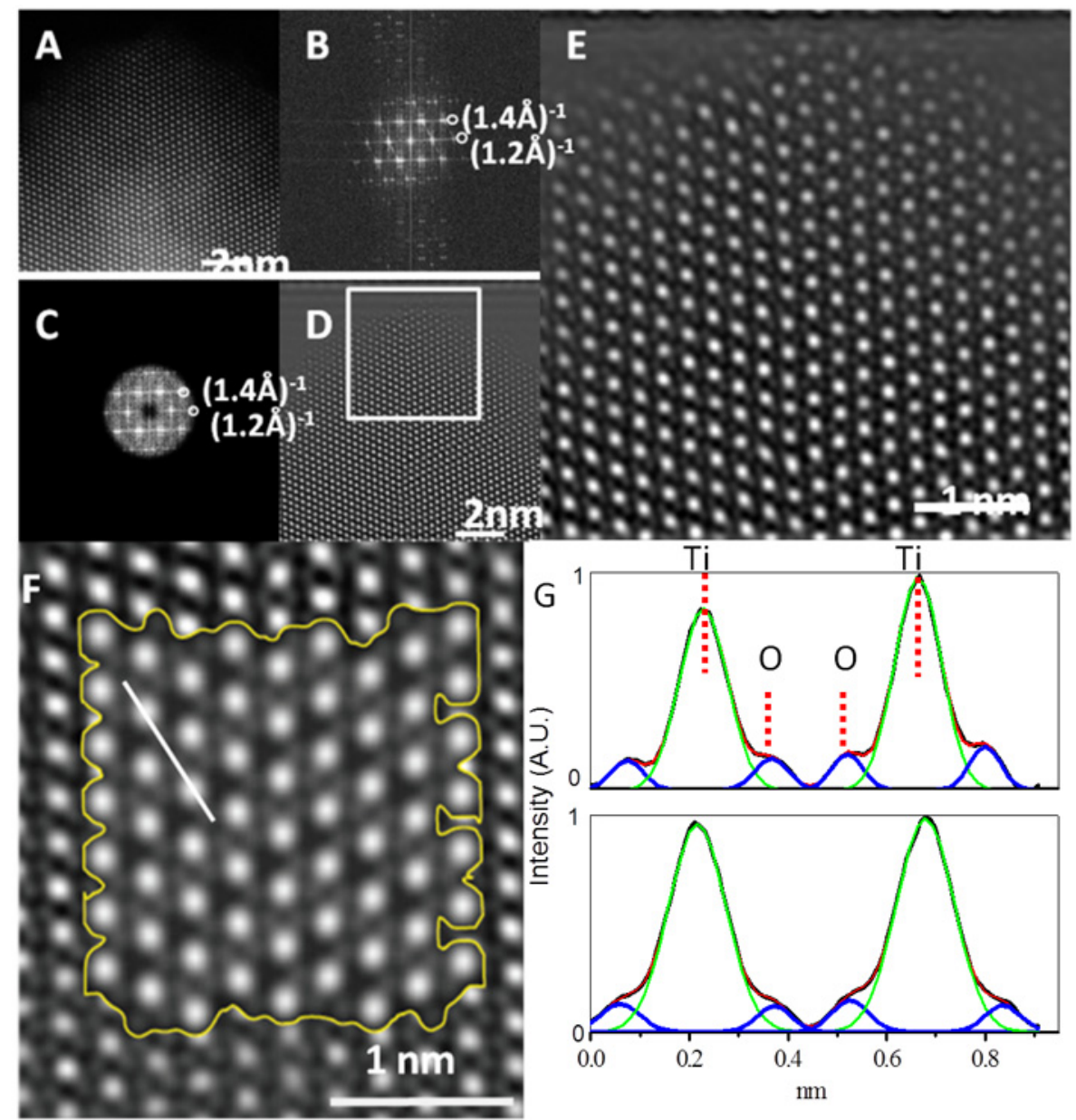

Figure 6. The process to enhance the oxygen column contrast by removing background noise. (A) The raw image of (101) twins, the intensity variation caused by thickness changes. (B) The FFT pattern of the raw HAADF image. (C) Periodic spots below $(1.4 \AA)^{-1}$ remained. (D) Reconstructed HAADF images from (A). (E) An enlargement of the area marked with a white rectangle in (D) shows enhanced oxygen column contrast. (F) The simulated image (inside the yellow periphery) imposed on the processed image. (G) Profile of the experimental image. $(\mathrm{H})$ Profile of the simulated image. The blue lines in $(\mathrm{G})$ and $(\mathrm{H})$ are the mathematical baselines for titanium and oxygen peak fitting.

For (301) twins, both the Ti and $\mathrm{O}$ atoms in the twin plane are mirror symmetric (figure 3(B)). The calculations conducted by Lee et al using an ionic shell model confirmed that the proposed atomic models are energetically favorable.

From the Wulff construction (figure S1(A) available at stacks.iop.org/Nano/23/335706/mmedia) plotted from calculated surface energies of different surfaces, $\{110\}$ is the predominate facet in equilibrium rutile crystal [38]. For rutile nanotwins, the corners of $\{110\}$ facets are truncated by $\{010\}$ facets, and the TEM image is the projection of the nanotwins along the $\langle 010\rangle$ direction (see figures S1(B) and (C) available at stacks.iop.org/Nano/23/335706/mmedia) as the nanorods rest on the TEM grids due to their unique V-shaped geometry. In the rutile unit cell, each titanium atom is coordinated with six oxygen atoms (figure $3(\mathrm{C})$ ). There is a distortion in the $\mathrm{TiO}_{6}$ octahedron: the axial bond lengths $(1.98 \AA)$ are slightly longer than that along the equatorial (1.95 $\AA$ ). In the projection along the [010] zone axis, each titanium row is surrounded by six oxygen columns (figure 3(D)). The distance between the two closest oxygen columns is $1.79 \AA$ and the closest distance between titanium rows and oxygen columns is $1.4 \AA$.

In the HAADF image of (101) twins, the main features are the bright dots contributed by $\mathrm{Ti}$ rows. The position of the twin boundary is clearly identified (indicated with a white arrow in figure 4(A)). The FFT pattern confirms the perfect symmetry at atomic level of the twin boundary. In the bright field image (figures 4(B) and (D)), the titanium rows are revealed as larger dark spots and the oxygen rows as small gray spots. At a higher resolution in the HAADF image, the oxygen columns can be practically resolved in the positions between titanium rows although their contrasts are much weaker than those of the titanium rows (indicated with arrows in figure $4(C)$ ). In the enlarged bright field image, both titanium rows and oxygen columns can be clearly resolved (figure 4(C)).

Similarly, both titanium rows and oxygen columns can be resolved in an HAADF image of (301) twins (figures 5(A) and (C)) as well as in the BF images (figures 5(B) and (D)) collected simultaneously with the HAADF images. 

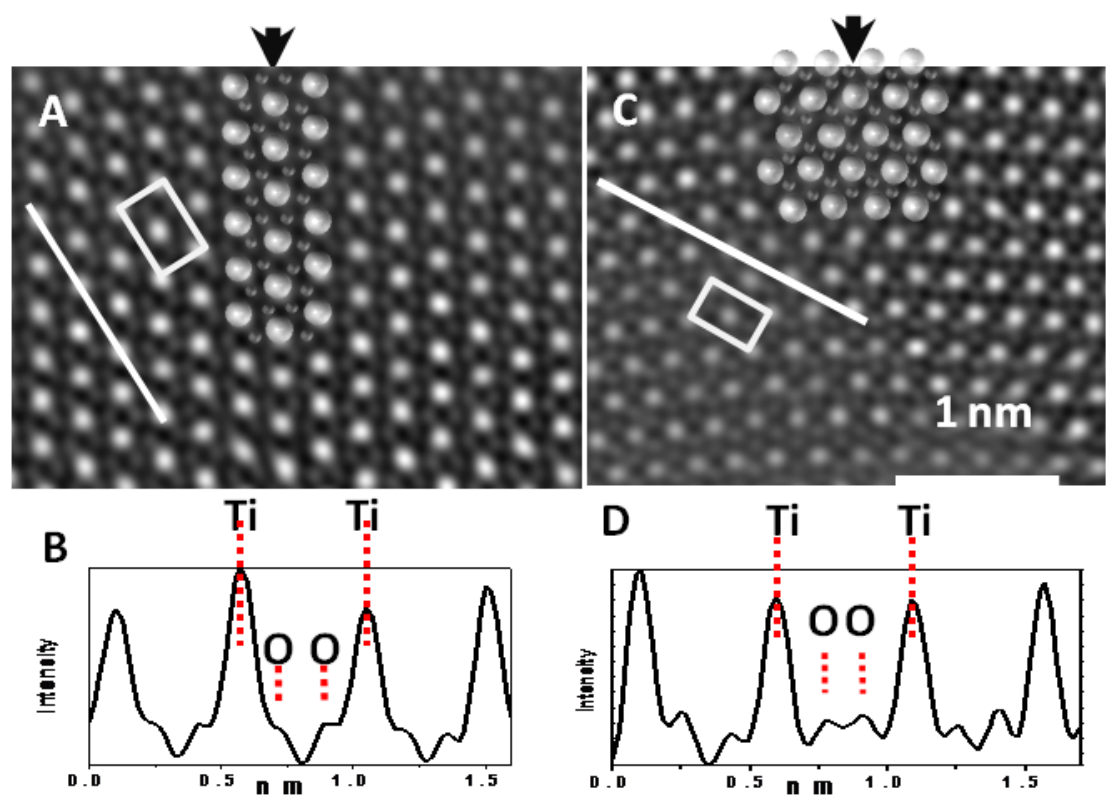

Figure 7. Processed HAADF images of rutile (101) and (301) twins. (A) For the (101) twins, the brighter features correspond to Ti rows, the less bright features are $\mathrm{O}$ rows, and the intensity profile aligns with the white line. The arrow indicates the position of the (101) twin boundary. (B) The intensity of the profile line shows the distances between titanium and oxygen rows in image (A). (C) For the (301) twins, the arrow indicates the position of the (301) twin boundary. (D) The intensity of the line profile shows the distances between the titanium and oxygen rows in image $(\mathrm{C})$.

The positions of titanium rows and oxygen columns of (101) and (301) twins can be resolved using the bright field images. In the corresponding HAADF images, the positions of oxygen columns can be practically resolved in some areas.

Figure 6(A) shows an HAADF-STEM raw image of a (101) twin joint; the intensity variation is mainly due to the variation in sample thickness caused by faceting in rutile nanotwins. A radial difference filter was applied to remove the background noise from the original HAADF images of the (101) twin boundary (figure 6) [39, 40]. Since the closest distance between titanium rows and oxygen columns is $1.4 \AA$, in the FFT image, information below $(1.4 \AA)^{-1}$ remained (figures $6(\mathrm{~B})$ and $\left.(\mathrm{C})\right)$. The contrast of oxygen columns was significantly enhanced by lowering the noise level (figure 6(E)). The simulated image (figure $6(\mathrm{H})$ ) reproduced the experimental image (figure $6(\mathrm{G})$ ) well; the large peaks are from titanium rows, with shoulders on the curve from oxygen columns. The signal to noise ratio of the oxygen columns has a significant enhancement with the same background removal process (figure S2 available at stacks.iop. org/Nano/23/335706/mmedia).

Figure 7 presents the processed HAADF images of the (101) and (301) twins. For the (101) twins (figures 7(A) and (B)), the average distance between $\mathrm{Ti}$ rows is $4.43 \AA$; the shoulders on the curve are from oxygen columns and their average distance is $1.73 \AA$. They are smaller than both the distance between Ti rows (4.59 $\AA$ ) and that between O rows $(1.79 \AA)$ in bulk rutile crystals (figure $3(\mathrm{D}))$. The unit cell in the twin boundary vicinity measured from the images is $2.87 \AA \times 4.43 \AA$. In the (301) twins, the distance between titanium rows is $4.56 \AA$; the distance between oxygen columns is $1.77 \AA$ (figures 7(C) and (D)). The unit cell in the vicinity of the (301) twin boundary measured from the image is
$2.88 \AA \times 4.56 \AA$. Both unit cell values are consistent within the error with the bulk lattice parameters. The data measured from the corresponding bright field images for the (101) and (310) twins (figure S3 available at stacks.iop.org/Nano/23/335706/ mmedia) have the same values as the data measured from HAADF images.

The HAADF image contrast is also affected by the sample's thickness due to the longitudinal coherence difference along the atomic columns, the probe channeling effect and probe intensity transfer between atomic columns [41-46]. As shown in figure S1 (available at stacks.iop.org/Nano/23/ $335706 /$ mmedia), the surfaces of rutile twins are faceted, the thickness along the twin boundary equals the projection of the diamond shape of (011) facets, the thickness along the twin boundary first increases and then decreases. According to figure S4 (available at stacks.iop.org/Nano/23/335706/ mmedia), the thickness across the twin boundaries first decreases, then increases, because the thickness across the twin boundary first equals the distance between (110) and (110) facets, then the distance equals the distance between (101) and (101) facets. The unique continuous variation in thickness structure provides an ideal example to study the relationship between HAADF images and sample thickness changes. In the processed HAADF images of the (101) and (301) twin boundaries (figure 8 and figure S5 available at stacks.iop.org/Nano/23/335706/mmedia), it is clear that oxygen rows can only be distinguished within a certain area in the vicinity of the twin boundary. According to the line profile across the twin boundary (in figure S4(B) available at stacks.iop.org/Nano/23/335706/mmedia), the twin plane is the thinnest place across the vicinity of the twin boundary. The appropriate thickness to visualize oxygen in the rutile twin boundary is less than $8 \mathrm{~nm}$. As the thickness increases 


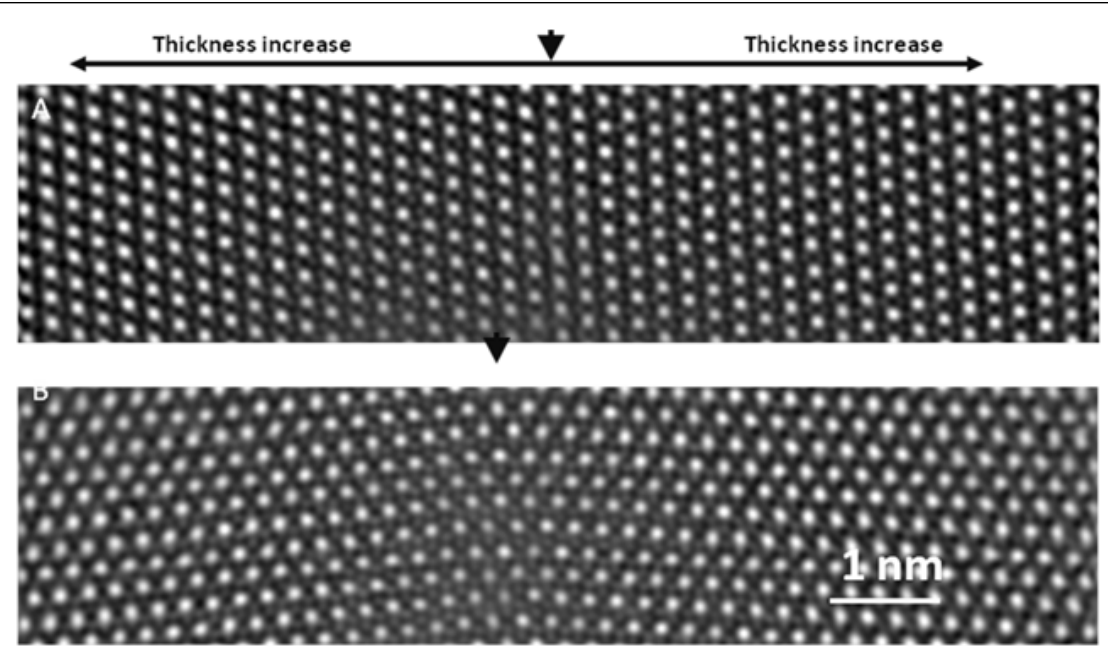

Figure 8. Processed HAADF images of the (101) and (301) twins across the twin boundaries for (A) (101) twins, and (B) (301) twins. The brighter features correspond to Ti rows and the less bright features are O rows. The arrows indicate the positions of the (101) and (301) twin boundaries. For both (101) and (301) twins, oxygen columns are more distinctive in the vicinity of the twin boundary.

across or along the twin boundary, the increased background noise totally conceals the relatively weak signals of oxygen columns.

These observations prove that the oxygen atoms in rutile twin boundaries can be directly resolved by using HAADF as well as bright field imaging. With the known oxygen and titanium column locations from both HAADF and BF images, the atomic structures of the rutile (101) and (301) twin boundaries were resolved directly from both HAADF and BF images (figures 5 and 6). In (101) twins, the twin plane consists of $\mathrm{Ti}$ atoms shared by two twin domains, and the sublattices of $\mathrm{Ti}$ atoms are mirror symmetric. The oxygen atoms around the Ti atoms are not mirror symmetric, but have $1 / 2\langle 111\rangle$ displacement from the mirror symmetric position. For (301) twins, both the $\mathrm{Ti}$ and the $\mathrm{O}$ atoms in the twin plane are mirror symmetric. Our observations are consistent with the atomic structures of (101) twins and (301) twins proposed from theoretical calculation and conventional HRTEM [35-37], in which the positions of oxygen rows could not be determined because the lattice images are dominated by metal columns.

In conclusion, the atomic structures of rutile and anatase nanocrystals were studied using $\mathrm{HAADF}$ and $\mathrm{BF}$ imaging modes; $\mathrm{BF}$ imaging is more powerful in imaging oxygen atomic columns than the HAADF mode. The positions of the oxygen columns in two kinds of rutile twin boundary were directly resolved using HAADF as well as bright field images. The ability to locate the positions of atomic oxygen columns in the twin boundaries allows for a direct determination of their atomic structures. The observed structures are consistent with previously proposed models in which the positions of the oxygen lattices were unknown.

\section{Acknowledgments}

The authors acknowledge funding and support from Baylor University (PJF), the ACS PRF Grant No. 51921-ND3 (PJF), and the NSF PREM Grant No. DMR 0934218 (MJY), title:
Oxide and metal nanoparticles - the interface between life sciences and physical sciences.

\section{References}

[1] Lu W, Bruner B, Casillas G, He J, Jose-Yacaman M and Farmer P J 2012 Large scale synthesis of V-shaped rutile twinned nanorods CrystEngComm 14 3120-4

[2] Gratzel M 2001 Photoelectrochemical cells Nature $414338-44$

[3] Fujishima A and Honda K 1972 Electrochemical photolysis of water at a semiconductor electrode Nature $\mathbf{2 3 8} 37$

[4] Oregan B and Gratzel M 1991 A low-cost, high-efficiency solar-cell based on dye-sensitized colloidal $\mathrm{TiO}_{2}$ film Nature $24353737-40$

[5] Zhang Z, Rousseau R, Gong J, Kay B D and Dohnalek Z 2009 Imaging hindered rotations of alkoxy species on $\mathrm{TiO}_{2}(110)$ J. Am. Chem. Soc. 131 17926-32

[6] Zhang Z et al 2008 Vacancy-assisted diffusion of alkoxy pecies on rutile $\mathrm{TiO}_{2}(110)$ Phys. Rev. Lett. 101156103

[7] Diebold U 2003 The surface science of titanium dioxide Surf. Sci. Rep. 48 53-229

[8] Penn R and Banfield J 1999 Morphology development and crystal growth in nanocrystalline aggregates under hydrothermal conditions: insights from titania Geochim. Cosmochim. Acta 63 1549-57

[9] Penn R and Banfield J 1999 Formation of rutile nuclei at anatase $\{112\}$ twin interfaces and the phase transformation mechanism in nanocrystalline titania Am. Mineral. 84 871-6

[10] Penn R and Banfield J 1998 Imperfect oriented attachment: dislocation generation in defect-free nanocrystals Science 281 969-71

[11] Erni R, Rossell M D, Kisielowski C and Dahmen U 2009 Atomic-resolution imaging with a sub-50-pm electron probe Phys. Rev. Lett. 102096101

[12] Sawada H et al 2009 STEM imaging of 47-pm-separated atomic columns by a spherical aberration-corrected electron microscope with a $300 \mathrm{kV}$ cold field emission gun J. Electron Microsc. 58 357-61

[13] Urban K W 2009 Is science prepared for atomic-resolution electron microscopy? Nature Mater. 8 260-2

[14] Dellby N, Krivanek O, Nellist P, Batson P and Lupini A 2001 Progress in aberration-corrected scanning transmission electron microscopy J. Electron. Microsc. 50 177-85 
[15] Bar Sadan M, Houben L, Enyashin A N, Seifert G and Tenne R 2008 Atom by atom: HRTEM insights into inorganic nanotubes and fullerene-like structures Proc. Natl Acad. Sci. 105 15643-8

[16] Isabell T et al 2009 Development of a $200 \mathrm{KV}$ atomic resolution analytical electron microscope Microsc. Today $178-11$

[17] Haider M, Uhlemann S, Schwan E, Rose H, Kabius B and Urban K 1998 Electron microscopy image enhanced Nature 392768

[18] Yoshida K, Kawai T, Nambara T, Tanemura S, Saitoh K and Tanaka N 2006 Direct observation of oxygen atoms in rutile titanium dioxide by spherical aberration corrected high-resolution transmission electron microscopy Nanotechnology 17 3944-50

[19] Shibata N et al 2008 Direct imaging of reconstructed atoms on $\mathrm{TiO}_{2}(110)$ surfaces Science 322 570-3

[20] Park K T, Pan M H, Meunier V and Plummer E W 2006 Surface reconstructions of $\mathrm{TiO}_{2}(110)$ driven by suboxides Phys. Rev. Lett. 96226105

[21] Findlay S D, Shibata N, Sawada H, Okunishi E, Kondo Y and Ikuhara Y 2010 Dynamics of annular bright field imaging in scanning transmission electron microscopy Ultramicroscopy 110903

[22] De Caro L, Carlino E, Caputo G, Cozzoli P D and Giannini C 2010 Electron diffractive imaging of oxygen atoms in nanocrystals at sub-angstrom resolution Nature Nanotechnol. 5 360-5

[23] Ishizuka K 2002 A practical approach for STEM image simulation based on the FFT multislice method Ultramicroscopy 90 71-83

[24] Heidenreich R D 1964 Fundamentals of Transmission Electron Microscopy (New York: Wiley-Interscience)

[25] Findlay S D, Azuma S, Shibata N, Okunishi E and Ikuhara Y 2011 Direct oxygen imaging within a ceramic interface, with some observations upon the dark contrast at the grain boundary Ultramicroscopy 111285

[26] Jia C L, Lentzen M and Urban K 2003 Atomic-resolution imaging of oxygen in perovskite ceramics Science $299870-3$

[27] Urban K W, Jia C L, Houben L, Lentzen M, Mi S B and Tillmann K 2009 Negative spherical aberration ultrahigh-resolution imaging in corrected transmission electron microscopy Phil. Trans. A 367 3735-53

[28] Krivanek O L et al 2010 Atom-by-atom structural and chemical analysis by annular dark-field electron microscopy Nature 464 571-4

[29] Huang R et al 2011 Oxygen-vacancy ordering at surfaces of lithium manganese (III, IV) oxide spinel nanoparticles Angew. Chem. Int. Edn 50 3053-7
[30] Oshima Y et al 2010 Direct imaging of lithium atoms in $\mathrm{LiV}_{2} \mathrm{O}_{4}$ by spherical aberration-corrected electron microscopy J. Electron Microsc. 59 457-61

[31] Huang $\mathrm{R}$ et al 2010 Real-time direct observation of $\mathrm{Li}$ in $\mathrm{LiCoO}_{2}$ cathode material Appl. Phys. Lett. 98051913

[32] Findlay S D et al 2010 Direct imaging of hydrogen within a crystalline environment Appl. Phys. Express 3116603

[33] Ishikawa R, Okunishi E, Sawada H, Kondo Y, Hosokawa F and Abe E 2011 Direct imaging of hydrogen-atom columns in a crystal by annular bright-field electron microscopy Nature Mater. 10 278-81

[34] Daneu N, Schmid H, Recnik A and Mader W 2007 Atomic structure and formation mechanism of (301) rutile twins from Diamantina (Brazil) Am. Miner. 92 1789-99

[35] Lee W Y, Bristowe P D, Gao Y and Merkle K L 1993 The atomic structure of twin boundaries in rutile Phil. Mag. Lett. 68 309-14

[36] Suzuki K, Ichihara M and Takeuchi S 1991 High-resolution electron microscopy of lattice defects in $\mathrm{TiO}_{2}$ and $\mathrm{SnO}_{2}$ Phil. Mag. A 63 657-65

[37] Gao Y, Merkle K L, Chang H L, Zhang T J and Lam D J 1992 Study of defects and interfaces on the atomic scale in epitaxial $\mathrm{TiO}_{2}$ thin films on sapphire Phil. Mag. A 65 1103-25

[38] Ramamoorthy M, Vanderbilt D and King-Smith R D 1994 First-principles calculations of the energetics of stoichiometric $\mathrm{TiO}_{2}$ surfaces Phys. Rev. B 49 16721-7

[39] Kilaas R 1998 Optimal and near-optimal filters in high-resolution electron microscopy J. Microsc. 190 45-51

[40] Ishizuka K, Eilers P and Kogure T 2007 Optimal noise filters in high-resolution electron microscopy Microsc. Microanal. 13 (Supplement $\mathrm{SO}_{2}$ ) 902-3

[41] Spence J C H 2003 High-Resolution Transmission Electron Microscopy 3rd edn (Oxford: Oxford University Press)

[42] Klenov D O and Stemmer S 2006 Contributions to the contrast in experimental high-angle annular dark-field images Ultramicroscopy 106889

[43] Loane R F, Xu P and Silcox J 1992 Incoherent imaging of zone axis crystals with ADF STEM Ultramicroscopy 40121

[44] Hillyard S, Loane R F and Silcox J 1993 Annular dark-field imaging: resolution and thickness effects Ultramicroscopy 4914

[45] Allen L J, Findlay S D, Oxley M P and Rossouw C J 2003 Lattice-resolution contrast from a focused coherent electron probe. Part I Ultramicroscopy 9647

[46] Rossouw C J, Allen L J, Findlay S D and Oxley M P 2003 Channelling effects in atomic resolution STEM Ultramicroscopy 96299 